\title{
Warum Antike?
}

\section{Missbrauch und Legitimation in Geschichte und Gegenwart}

\section{Alte Sprachen: ein elitärer Popanz?}

Die Alten Sprachen sind ein Bildungsgegenstand, der seit langer Zeit umkämpft ist. Hier geht es nicht in erster Linie um Nutzen oder Nutzlosigkeit; es geht vor allem auch um Ideologie. Die Beschäftigung mit den Argumentationen pro und contra Altsprachenunterricht ist historisch sehr aufschlussreich - wobei sich die Kenntnis der Materie als unerlässlich erweist, um tendenziöse Interpretationen offenzulegen. Anders als die überwiegende Zahl der anderen in diesem Band versammelten Beiträge befasst sich dieser Aufsatz primär mit den Fächern Latein und Griechisch. Diese stehen jedoch in unmittelbarem Zusammenhang mit historischem Lernen, insofern die Auseinandersetzung mit Originaltexten wichtige Quellenkompetenzen vermittelt.

Gerade in letzter Zeit ist der altsprachliche Unterricht wieder einmal in die Schlagzeilen geraten: In der Tagespresse wurde vielfach über ein DFG-Projekt des Soziologen Jürgen Gerhards von der Freien Universität Berlin (FU) berichtet, der „Die Wahl von Latein und Altgriechisch als schulische Fremdsprachen: Eine Distinktionsstrategie der oberen sozialen Klassen" untersucht. ${ }^{1}$ Die zentrale Annahme des Projekts lautet, dass die „mit kulturellem Kapital privilegierten Klassen [...] durch die Wahl von Latein und Altgriechisch Distinktions- und Exklusionsgewinne erzielen [wollen]" und die weniger privilegierten Klassen diese Verhaltensweise kopieren, sich aber stärker an einer Verwertungslogik orientieren und sich positive Transfereffekte erhoffen. ${ }^{2}$ Sind die Alten Sprachen also nur ein Popanz bildungsbürgerlicher Traditionsversessenheit? Natürlich ist diese Reduzierung der Motivation auch ein politischer Angriff auf den vermeintlich elitären Altsprachenunterricht, der soziale Gräben verbreitert und Jugendliche mit anderen Bildungsbiografien benachteiligt.

\footnotetext{
1 https://gepris.dfg.de/gepris/projekt/321602695, vgl. z. B. Heike Klovert, Mythos und Wirklichkeit: „Wer in der Schule Latein hatte, gilt als höher gebildet", in: Der Spiegel (2.9.2019), https://www.spiegel.de/lebenundlernen/schule/latein-in-der-schule-warum-die-sprache-im-lehrplan-kaum-sinn-ergibt-a-1284513.html (letzter Zugriff: 26.5.2021), oder Jürgen Gerhards im Gespräch mit Thekla Jahn, Diskussion um „tote Sprache“" Es macht wenig Sinn, heute noch Latein zu lernen", in: Deutschlandfunk (27.8.2019), https://www.deutschlandfunk.de/diskussion-um-tote-sprache-es-macht-wenig-sinn-heute-noch.680.de.html?dram:article_ id=457388 (letzter Zugriff: 26.5.2021).

2 https://www.polsoz.fu-berlin.de/soziologie/arbeitsbereiche/makrosoziologie/projekte/proj_latein-altgriechisch.html (letzter Zugriff: 26.5.2021).

> Das Werk ist unter der Creative-Commons-Lizenz Namensnennung - Weitergabe unter gleichen Bedingungen 4.0 International veröffentlicht. Den Vertragstext finden Sie unter: https://creativecommons.org/licenses/by-sa/4.0/deed.de. Bitte beachten Sie, dass einzelne, entsprechend gekennzeichnete Teile des Werks von der genannten Lizenz ausgenommen sein bzw. anderen urheberrechtlichen Bedingungen unterliegen können.
} 
Dass der altsprachliche Unterricht im Kern elitär ist, kann nicht ernsthaft bestritten werden. Es gibt aber seit Langem Versuche, Barrieren zu durchbrechen, z. B. das Projekt Pons Latinus der Humboldt-Universität Berlin (HU). In einer Partnerschulkoordination der HU mit einer Neuköllner Schule mit sehr hohem Migrations-Anteil wird seit Jahren erfolgreich Latein unterrichtet; diverse Studien belegen, dass die Jugendlichen mit nichtdeutschen Herkunftssprachen ihre Deutschkompetenzen steigern und dies auch selber positiv empfinden. ${ }^{3}$

Die Lösung dieser Frage wird wohl sein, dass Sprachkompetenzen durch gezielte sprachvergleichende Aufgabenstellungen geschult werden, wie sie der Lateinunterricht mit seinen Legitimationsnöten allmählich mehr als andere Sprachfächer zu bieten beginnt. Freilich nutzen die Lehrpersonen, die sich einer sprachkompetenz-orientierten Lateindidaktik bedienen, auch die Neuköllner Gruppe, nicht einfach herkömmliche Unterrichtsmaterialien, sondern wenden im Latein-Unterricht etwa Konzepte aus der DaZDidaktik an. Die Kompetenzen, die hier erworben werden, könnten durch dieselben Aufgabenstellungen auch in neuphilologischen Fächern geschult werden könnten. In jedem Fall argumentieren beide Seiten politisch: Latein (und Griechisch) ist nicht einfach irgendein Fach, sondern steht zu Recht oder zu Unrecht für gesellschaftliche Veränderung.

\section{Antike und Politik}

Dass die Alten Sprachen zu ihren Gunsten oder Ungunsten politisch instrumentalisiert werden, ist nicht neu. ${ }^{4}$ Die Diskussion um den Sinn von Latein und Griechisch an der Schule läuft im Grunde genommen, seitdem Latein gesellschaftlich nicht mehr als aktiv beherrschte Sprache vorausgesetzt wurde. Wilhelm von Humboldts preußisches Gymnasium sieht Latein und Griechisch als fest verankerte Bildungssprachen ohne unmittelbaren praktischen Anwendungsbezug vor: Latein war immerhin noch die übliche Wissenschaftssprache, Griechisch diente als Grundlage für das Studium einer von Humboldt idealisierten Gesellschaft. Die Arbeitsenergie der Schüler*innen, die in das Erlernen der Alten Sprachen gesteckt wurde, war nach den preußischen Gymnasialreformen nicht geringer als hundert Jahre zuvor, wo Latein noch gesprochen wurde. Dies kann als entscheidender Wendepunkt gelten: „Paradoxerweise bedeutete gerade die faktische Kontinuität der Stundenverteilung im Lehrplan einen Paradigmenwechsel der Bildungs-

\footnotetext{
3 Z. B. Carola Fengler, Lateinunterricht und ausländische Schüler - ein Erfahrungsbericht, in: PegOn 1, 2000, 1-12, http://www. pegasus-onlinezeitschrift.de/alte_seite/erga12000fengler.htm (letzter Zugriff: 26.5.2021); Stefan Kipf, Integration durch Bildung. Schülerinnen und Schüler nichtdeutscher Herkunftssprache lernen Latein, in: Forum Classicum 53, 2010, 3, 181-197, https://doi. org/10.11588/fc.2010.3.38974; Maria Große, Pons Latinus - Modellierung eines sprachsensiblen Lateinunterrichts, in: Info-DaF 1, 2014, 70-89, http://www.daf.de/downloads/InfoDaF_2014_Heft_1.pdf (letzter Zugriff: 26.5.2021).

4 Vgl. umfassend Anna Kranzdorf, Ausleseinstrument, Denkschule und Muttersprache des Abendlandes. Debatten um den Lateinunterricht in Deutschland 1920-1980. Berlin 2018
} 
vorstellung, wie er extremer kaum gedacht werden kann." Latein und Griechisch steigerten sogar ihre globale Präsenz, indem das deutsche Gymnasium international kopiert und die Alten Sprachen überall in der europäisch dominierten Welt gelehrt wurden.

Humboldts Argumentation für das Studium der Antike, dargelegt vor allem in seiner 1873 verfassten Schrift Über das Studium des Alterthums, und des Griechischen insbesondere, muten heutzutage abenteuerlich an. In diesem ,Gründungsdokument des altsprachlichen Unterrichts in der Neuzeit' legt er zum Beispiel dar, dass nicht jede Nation in gleicher Weise geeignet sei, sich mit ihr zu befassen und damit Menschenkenntnis zu erlangen, da nicht jede Nation in gleicher Weise vielseitig und authentisch sei bzw. repräsentativ für die Menschheit als solche. Vor allem das antike Griechenland erfüllt nach Humboldt diese Voraussetzungen. Davon abgesehen, dass Humboldts Vorstellungen von unter- und überlegenen Kulturen anachronistisch erscheinen, erweisen sich seine Darlegungen auch im Detail als schwer nachvollziehbar, so zum Beispiel, dass die von ihm postulierte stark ausgebildete nationale Identität der Griechen durch Isolation zustande gekommen sei: „Nun waren die Griechen mit keinem einzigen höher gebildeten Volke vor

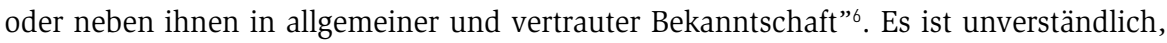
wie Humboldt zu so einer Einschätzung kommen konnte; so hatte die Indogermanistik zu diesem Zeitpunkt schon zahlreiche Übereinstimmungen zwischen dem Griechischen und etwa Sanskrit nachgewiesen. Auch die ausführlichen ethnographischen Exkurse eines Herodot scheint Humboldt zu ignorieren, wenn er schreibt: „Die Geschichte ist grossentheils Griechische, und wo sie es auch nicht ist, sind wenigstens die früheren griechischen Geschichtschreiber noch zu wenig gewohnt, mehrere Völker zu vergleichen, und Eignes und Fremdes voneinander abzusondern, auch zu sehr mit allem Vaterländischen beschäftigt, als dass nicht sehr oft der Grieche durchblikken sollte."

Dass die Stellung, die Humboldt den Alten Sprachen einräumte, nicht unangefochten bleiben konnte, war eigentlich von Anfang an klar; notwendigerweise ergaben sich in Folge Legitimationsstrategien, die unweigerlich zu weiteren Problemen führten.

Wirkmächtige Kritik am altsprachlichen Gymnasium wurde achtzig Jahre nach den Preußischen Reformen von Wilhelm II. geübt: In einem Erlass von 1889 forderte er, die Schulen nutzbar zu machen, ,um der Ausbreitung sozialistischer und kommunistischer Ideen entgegenzuwirken”, worin die Schulen bis dato versagt hätten. ${ }^{8}$

5 Jürgen Leonhardt, Latein. Geschichte einer Weltsprache. München 2009, 260.

6 Wilhelm von Humboldt, Über das Studium des Alterthums, und des Griechischen insbesondere, Entstanden 1793; Erstdruck in: Sechs ungedruckte Aufsätze über das klassische Altertum von Wilhelm von Humboldt, herausgegeben von Albert Leitzmann, Leipzig $1896, \S 18$.

7 Ebd. §19.

8 Erlass Kaiser Wilhelms II. zur Reform des Schulunterrichts als Mittel zum Kampf gegen den Sozialismus (1. Mai 1889), http:// ghdi.ghi-dc.org/sub_document.cfm?document_id=1807\&language=german (letzter Zugriff: 26.5.2021). 
Auf einer Schulkonferenz im Jahr 1890 prägte er das Diktum, man solle „nationale junge Deutsche erziehen, und nicht junge Griechen und Römer". ${ }^{9}$

Die Lehrenden reagierten und förderten im Lateinunterricht fortan vorwiegend die Lektüre solcher Texte, die irgendwie nationalistisch verwertbar waren, z. B. die Germania des Tacitus und Caesars Bellum Gallicum. Die Griechen als ,Erfinder` der Demokratie rückten in den Hintergrund; nun galt die Monarchie als Errungenschaft der Antike, wie etwa der Althistoriker Robert Pöhlmann betont, der „soziales Königtum” zum Schutz für alle Bürger als Idee der Griechen bezeichnet und die Idee der konstitutionellen Monarchie bei Platon und Aristoteles angelegt sieht. ${ }^{10}$

Groteske Blüten trieb die Anbiederung an den Zeitgeist im Nationalsozialismus. Führende NS-Ideologen zogen die Antike zunächst auch ohne Hilfe der klassischen Philologie als zentrales Paradigma heran; Adolf Hitler identifizierte sich stark mit der griechisch-römischen Antike, die ihm als Legitimationsinstanz für seine Weltherrschaftspläne diente; er sah sich in einem universalhistorischen Rahmen und erinnerte in seinen Reden an zahlreiche antike Potentaten. Hitler verlangte, den Geschichtsunterricht zu reduzieren; nicht Zahlen und Fakten sollten im Vordergrund stehen, sondern „Erkennen der großen Entwicklungslinien”, eine Weltgeschichte, „in der die Rassenfrage zur dominierenden Stellung erhoben wird."11 Hierbei steht der Gegensatz zwischen Kulturbegründern und Kulturzerstörern im Vordergrund; alle aus Hitlers Sicht kulturbegründenden Kulturen waren „arisch” (z. B. die Griechen), alle kulturzersetzenden „semitisch” (z. B. Karthago). Hitlers vollmundige Behauptungen sind auch in den sogenannten „Tischgesprächen” belegt: So sei „der Germane” in den Süden ausgewandert, um dort in einem angenehmeren Klima seine Fähigkeiten voll ausbilden zu können. ${ }^{12}$ Diese kruden Ideen waren nicht allein Hitlers Erfindung; dahinter standen diverse einflussreiche Theoretiker wie Houston Stewart Chamberlain, Alfred Rosenberg oder Richard Walther Darré. Entscheidend ist, dass das Griechen- und Römertum zum reinen Symbolfaktor geworden war; genaues Hinsehen war nicht erwünscht. An echter Antikenkompetenz bestand kein Interesse.

Genau dies ist heute wieder der Fall, wo die Antike und ihr Erbe erneut von Rechtspopulisten missbraucht wird. In einem gerade viel beachteten Buch weist dies Donna Zuckerberg an verschiedenen Paradigmen nach, die sich überwiegend um das Gender-

9 „Die Antike hat in einem nationalistisch erstarkenden Deutschland keinen Platz mehr. Wer selber auf dem Gymnasium gewesen ist und hinter die Kulissen gesehen hat, der weiß, wo es da fehlt. Und da fehlt es vor allem an der nationalen Basis. Wir müssen als Grundlage für das Gymnasium das Deutsche nehmen; wir sollen nationale junge Deutsche erziehen, und nicht junge Griechen und Römer. Wir müssen von der Basis abgehen, die jahrhundertelang bestanden hat, von der alten klösterlichen Erziehung des Mittelalters, wo das Lateinische maßgebend war und ein bißchen Griechisch dazu. Das ist nicht mehr maßgebend. Wir müssen das Deutsche zur Basis machen. Der deutsche Aufsatz muß der Mittelpunkt sein, um den sich alles dreht." Rede Wilhelms II. auf Schulkonferenz von 1890, in: Verhandlungen über Fragen des höheren Unterrichts. Berlin, 4.-17. Dezember 1890. Im Auftrage des Ministers der geistlichen, Unterrichts- und Medizinal-Angelegenheiten. Berlin 1891, $72 \mathrm{f}$.

10 Robert Pöhlmann, Aus Altertum und Gegenwart. Gesammelte Abhandlungen. München 1895, 4f.

11 Christian Hartmann/Thomas Vordermayer/Othmar Plöckinger/Roman Töppel (Hrsg.), Hitler, Mein Kampf. Eine kritische Edition, Bd. 2. München/Berlin 2016, $1071 \mathrm{f}$.

12 Hitlers, Tischgespräche', aufgez. von Heinrich Heims 1941-1944, Wolfsschanze 4.2.1942, in Der Spiegel 8, 1964, https://www. spiegel.de/spiegel/print/d-46163235.html (letzter Zugriff: 26.5.2021). 
Bild der US-amerikanischen neuen Rechten drehen. Es geht um die sogenannten ,Red Pillers', also Online-Communities, die sich dem Aufdecken von vermeintlich verschleierten Wahrheiten widmen wollen. Zuckerberg zeigt, wie sich misogyne Gruppen auf antike Texte beziehen, die sie sehr wörtlich und selbstverständlich ohne jede literaturwissenschaftliche oder historisch-kritische Kompetenz lesen. So werden stoische Texte, etwa Epiktets Encheiridion oder Marc Aurels Meditationes, als Männlichkeitstraining verkauft, um durch typisch männliche Vernunftbegabung emotionalen Stress zu überwinden, und Ovids Ars amatoria wird zum praktikablen Leitfaden für das effiziente und emotionslose ,Aufreißen` von Frauen heruntergebrochen. Zuckerberg konzentriert sich auf diese Gender-Aspekte, äußert sich aber auch über den Missbrauch antiker Inhalte zur Konstruktion einer weißen, europäischen Identität, so etwa durch die US-amerikanische Gruppe Identity Evropa. Auch die deutsche identitäre Bewegung bedient sich bei antiker Symbolik; so ist ihr Lambda-Label den spartanischen Schilden nachempfunden, wie sie in Frank Millers und Lynn Varleys Comic 300 (1998) bzw. im darauf basierenden Spielfilm von Zack Snyder (2006) dargestellt werden. Sparta wird in diesem Kontext als europäische Bastion verstanden, die die eigene Kultur gegen den andrängenden Orient in Form des persischen Heeres verteidigt.

Griechenland, Rom, Europa, westliche Zivilisation - diese Begriffe werden undifferenziert vermengt und populistisch eingesetzt. Dass dies schon lange der Fall ist und desaströse Folgen gezeitigt hat, hat kürzlich die US-amerikanische Society for Classical Studies in einem Statement zur Black Lives Matter-Bewegung formuliert:

„The Society for Classical Studies recognizes and acknowledges the complicity of Classics as a field in constructing and participating in racist and anti-black educational structures and attitudes. [...] On the one hand, white supremacist and nationalist groups have misappropriated Classics and other pre-modern fields for their own hateful agendas. On the other, within the discipline and profession itself scholars have perpetuated racist attitudes and ideas. These attitudes and ideas include but are not limited to the misleadingly reductive notion of a 'Western Civilization' resulting from an allegedly linear transfer of knowledge from Greece to Rome to Western Europe; the whitewashing of ancient Mediterranean culture; the uncritical use of Greece and Rome as ideals [...]."13

\section{Lesen lohnt sich}

Gegen diesen Missbrauch der Antike als populistisches Symbol hilft nur das, was im Nationalsozialismus verhindert werden und durch Konzentration auf die "großen Entwicklungslinien” ersetzt werden sollte: Genaues Hinsehen.

13 Statement on Police Brutality, Systemic Racism, and the Death of George Floyd. From the SCS Board of Directors, approved 6/3/20, https://classicalstudies.org/scs-news/from-scs-leadership (letzter Zugriff: 26.5.2021). 
Wahrscheinlich hat niemand von der Identitären Bewegung die Thermopylen-Geschichte im Original nachgelesen, nämlich bei Herodot (7.201-239). Wer das tut, findet zwar einen heldenhaften Leonidas, aber auch einen Haufen uneiniger Griechen, die zum großen Teil gar nicht dabei sind, als Europa vor dem Orient verteidigt werden soll; die Spartaner feiern das Fest der Karneien und haben deswegen nur die kleine Delegation um Leonidas losgeschickt, die anderen Griechen halten gerade die Olympischen Spiele ab und kreuzen auch nur mit einer Vorausabteilung auf. Andere griechische Städte haben längst mit den Persern kollaboriert. Die Geschichte ist auch nicht besonders pathetisch erzählt, indem Herodot gewohnt enzyklopädisch alle ihm bekannten Vorgeschichten, Parallelversionen, Gerüchte und Konsequenzen referiert.

Aber auch wenn an das ,echte' Heldentum des Leonidas und seiner Getreuen geht, lohnt sich ein Blick in den Originaltext. „Die Lakedaimonier kämpften rühmenswert und zeigten, daß sie das Kriegshandwerk verstanden, der Feind aber nicht” übersetzt Josef Feix einigermaßen martialisch (7.211.3); die deutschen Formulierungen finden sich aber im griechischen Original nicht alle wieder. Mit „,rühmenswert” hat Feix ảxi $\omega \varsigma \lambda$ ó $\gamma$ ov wiedergegeben, was eher ,erwähnenswert' bedeutet, und der markige kollektive Singular ,der Feind' findet sich im Griechischen gar nicht; Lakedaimonier und Perser werden

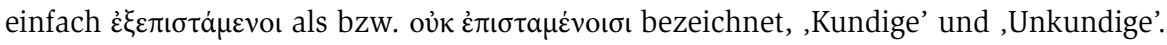
Das Wort ,Feind' kommt überhaupt nicht vor. Im Originaltext löst sich das meiste Pathos der Geschichte in Luft auf. An diesem Beispiel zeigt sich, wie sehr das Verständnis der Texte an der Originalsprache hängt - wenn dieses nicht gegeben ist, besteht immer eine Abhängigkeit von Vermittlungspersonen, die die Antike, wie sich gezeigt hat, mit Vorliebe als Untermauerung eigener Agenden missbrauchen.

Am Ende noch einmal zurück zu den aktuellen Argumentationen für oder gegen alte Sprachen: Beide Berliner Forschungsgruppen, die DFG-Studie der FU und das an der HU angesiedelte Projekt Pons Latinus, scheinen sich auf die Frage zuzuspitzen, ob der Altsprachenunterricht von echtem Nutzen sei oder sinnlose Symbolhandlung. Eine zusätzliche Dimension tut sich auf, wenn man eine intensive Beschäftigung mit der Antike als Dekodierung von Symbolen auffasst. Nadja Thoma hat schon 2015 postuliert, dass Latein und Griechisch nicht einfach Sprachen sind, deren Erlernen irgendwelche hilfreichen Sekundäreffekte zeitigt. Es ist vielmehr die Geschichtlichkeit der Fächer selbst, die viel aussagt über die Geschichte unserer Gesellschaft und deren Verständnis von Bildung, „wenn die Perspektivität in der Wahrnehmung von Geschichte, der Interpretation von Literatur und den darin zugrunde gelegten Menschenbildern zum konstitutiven Teil der Analyse gemacht wird und Schüler*innen und Lehrer*innen in diesem Sinne ihre eigene Involviertheit $[\ldots]$ in gesellschaftliche Verhältnisse mitreflektieren”. ${ }^{14}$

14 Nadja Thoma, Ein ,neutrales Vergleichsmedium, das niemandes Muttersprache ist'? Zur Bedeutung der ('Bildungs') Sprachen Latein und Griechisch in fachdidaktischen Diskursen der amtlich deutschsprachigen Migrationsgesellschaft, in: Nadja Thoma/ Magdalena Knappik (Hrsg.), Sprache und Bildung in Migrationsgesellschaften. Machtkritische Perspektiven auf ein prekarisiertes Verhältnis. Bielefeld 2015, 179-203, hier 196. 
Jeder kann alles behaupten über Nutzen und Schaden der antiken Sprachen und Paradigmen. Tatsache ist aber, dass diese Paradigmen kulturhistorisch von so großer Wirkmächtigkeit sind, dass wir uns kaum erlauben können, sie nicht genau zu kennen.

\section{Autorin}

\section{Katharina Wesselmann}

Professorin für Didaktik der Alten Sprachen an der Christian-Albrechts-Universität zu Kiel. Ihre Forschungsschwerpunkte sind antikes Epos und Geschichtsschreibung, narrative Topoi, mythische und rituelle Prägungen von Texten, Mehrsprachigkeitsdidaktik, Geschichte und Gegenwart des altsprachlichen Unterrichts.

kwesselmann@email.uni-kiel.de 